\title{
Decentralized Decisions and Technological Progress: A Formal Proof
}

\author{
Yong $\mathrm{TaO}^{\dagger}$ \\ College of Economics and Management, Southwest University, Chongqing, China
}

\begin{abstract}
This paper provides attempts to formalize Hayek's theory of knowledge. It has been theoretically shown that exponential income distribution is a spontaneous order of the well-functioning market economy. We show that this theoretical result is supported by the empirical evidence from the United Kingdom and China. In particular, we empirically show how the income structure of China evolved towards an exponential distribution after the market-oriented economic reformation. Furthermore, we strictly prove that, if the income structure of an economy obeys an exponential distribution, the income summation over all households leads to an aggregate production function with Hicks-neutral-like technical progress, in which the technology factor is exactly equal to society's information stock that is a result of combining all of decentralized decisions.
\end{abstract}

Keywords: Generalized Pareto distribution; Exponential income distribution; Technology factor; Information stock; Decentralized decisions

JEL Classification: O33; D31; D83; C46

$\dagger$ This work was supported by the Social Science Planning Project of Chongqing (Grant No. 2019PY40) and the Fundamental Research Funds for the Central Universities (Grant No. SWU2109209). Email address: taoyingyong@yahoo.com 


\section{Introduction}

The technology factor in aggregate production function is a mysterious variable. Distinguishing from labor and capital, Solow (1956) has used his famous model to show that the technology factor is the most powerful driver of economic growth. However, in his model, the technology factor is exogenously given. As a result, for the given technology, Solow's model predicts that, due to the diminishing marginal return of capital, the growth rate of per-capita income will decline to zero. This implies that, in the economic world described by Solow's model, the growth of per-capita income is unsustainable. To overcome the dilemma of Solow's model, motivated by Arrow's learning by doing (Arrow 1962), Lucas (1988), Romer (1986, 1990), and Barro (1990) endogenized the technology factor. Following their models, the technology factor will persistently rise with knowledge accumulation so that the growth rate of per-capita income is always higher than zero. These models are called the "endogenous growth models", in which the technology factor has been proposed as "knowledge" or “information stock" (Arrow, 1962; Saviotti, 1988; Jones, 1995; Romer, 2012; Acemoglu et al., 2016; Arthur, 2018; Nordhaus, 2021). In the 1990s, the conception of technology factor as "knowledge" or "information stock" was further used to explain the success of China's gradual reform of economic development ${ }^{1}$ (Murrell, 1992). Although the technology factor has been commonly interpreted as "knowledge" or "information stock", to date there is no formal proof for the validity of this interpretation. Our paper fills this gap. Here, we strictly prove that if the income structure of an economy obeys an exponential distribution, the technology factor in aggregate production function is exactly equal to society's information stock.

In the economic literature, it has been observed empirically that, for marketeconomy countries, the low- and middle-income class (about $90 \%$ of populations)

\footnotetext{
${ }^{1}$ Recently, Lin (2011) attempted to explain the success of China's gradual reform of economic development from the perspective of matching technological choice and factor endowment. This is in accordance with the conception of technology factor as "knowledge" or "information stock". In this regard, Murrell (1992) has pointed out that a society's stock of personal knowledge is acquired through a long historical process and is shaped by the institutions and organizations of that particular society that has the given factor endowment.
} 
follows an exponential distribution (Nirei and Souma, 2007; Newby et al., 2011; Clementi et al., 2012; Prinz, 2016; Irwin and Irwin, 2017; Rosser, 2019; Tao et al., 2019; Ma and Ruzic, 2020) and the top income class (less than 5\% of populations) follows a Pareto distribution (Mandelbrot, 1960; Nirei and Souma, 2007; Atkinson et al., 2011; Aoki and Nirei, 2017; Tao et al., 2019). Theoretically, it has been known that the generalized Pareto distribution is a fairly general family that includes the Pareto distribution and the exponential distribution as two special cases (Balkema and Haan, 1974; Pickands, 1975; Singh and Maddala, 1976; Cowell, 2000). Based on this theoretical result, Blanchet et al. $(2017,2018)$ used the generalized Pareto distribution to describe the income structure of the total population. In particular, the exponential distribution is a special case of the generalized Pareto distribution when the income structure turns to equal opportunity (Tao, 2021). This implies that the exponential distribution is expected to dominate the income structure of an equal-opportunity market economy, which indicates a well-functioning market economy. In the economic literature, a peer-to-peer economy without an intermediary third-party is recognized as an equal-opportunity market economy (Einav et al., 2016; Davidson et al., 2018; Filippi et al., 2020; Hojckova et al., 2020). In this vein, Tao (2016, 2021) used a long-run Arrow-Debreu economy (ADE), in which there are many households, each of whom independently operates a firm, to simulate a peer-to-peer economy that shares the publicly available technology. Furthermore, Tao $(2016,2021)$ showed that an exponential income distribution will emerge spontaneously in such a peer-to-peer economy. Based on this finding, Tao $(2016,2021)$ proposed to identify the exponential income distribution as the benchmark structure of the well-functioning market economy. As evidence, using a large sample, Tao et al. (2019) analyzed datasets of household income from 66 market-economy countries, ranging from Europe to Latin America, North America, and Asia, and found that, for all the countries, the income structure for the low and middle classes (about $90 \%$ of populations) uniformly follows an exponential distribution. Because the empirical evidence has shown that exponential income distribution dominates the vast majority of the populations, we assume that the income structure of the total population in a market-economy country is approximately 
described by an exponential distribution. Using this assumption, we strictly prove that the income summation over all households leads to an aggregate production function with Hicks-neutral-like technical progress, in which the technology factor is exactly equal to society's information stock.

This theoretical result may shed a light on understanding Hayek's theory of knowledge (Hayek, 1945, 1988). In this regard, Hayek (1988) argued:

"Thus, differences among individuals increase the power of the collaborating group beyond the sum of individual efforts... The knowledge that plays probably the chief role in this differentiation-far from being the knowledge of any one human being, let alone that of a directing superbrain-arises in a process of experimental interaction of widely dispersed, different and even conflicting beliefs of millions of communicating individuals. The increasing intelligence shown by man is, accordingly, due not so much to increases in the several knowledge of individuals but to procedures for combining different and scattered information which, in turn, generate order and enhance productivity... The importance and value of order will grow with variety of the elements, while greater order in turn enhances the value of variety, and thus the order human cooperation becomes indefinitely extensible" (Hayek, 1988, page 80).

In this argument, Hayek conceived an ideal society, in which, due to individual differentiation, decentralized decisions among individuals cause collective intelligence, which is reflected as an order of human cooperation, and in turn enhance productivity. In this order, there are information transmissions and transactions that are cost-free, and all of individuals will reach consensuses on resource allocation. All of these features indicate that the ideal society in Hayek's mind can be described by a peer-to-peer economy that shares the publicly available technology. Therefore, we can reexamine Hayek's theory of knowledge in the context of this peer-to-peer economy. Tao (2016, 2021) has shown that the exponential income distribution emerges spontaneously in such a peer-to-peer economy. As a spontaneous economic order, the exponential income distribution consists of a set of general equilibrium allocations among households (Tao, 
2016, 2021), in each of which all households reach consensuses on resource allocation. In the context of neoclassical economics, general equilibrium allocation is a collection of decentralized equilibrium decisions among households. Thus, in this paper we do not distinguish between general equilibrium allocation and decentralized equilibrium decisions. The main purpose of this paper is to show that, when the exponential income distribution emerges, the joint-event probability of decentralized decisions among households has an elegant relationship with the technology factor in aggregate production function, which can be written in the form of Shannon's information formula. This means that technological progress can be interpreted as a process of accumulating society's information stock, which depends on the combination of all of decentralized decisions.

The remainder of the paper is organized as follows. In section 2, we empirically show the validity of the exponential income distribution in describing the income structure of the well-functioning market economy. In section 3, we show that if the income structure of an economy obeys the exponential distribution, then the income summation over all households leads to a neoclassical aggregate production function with Hicks-neutral-like technical progress. In section 4, we further prove that the technology factor in this aggregate production function is exactly equal to society's information stock. Section 5 concludes the paper.

\section{Exponential income distribution}

For market-economy countries, it has been empirically observed that the income structure of top income class (less than $5 \%$ of populations) obeys a Pareto distribution (Mandelbrot, 1960; Nirei and Souma, 2007; Atkinson et al., 2011; Aoki and Nirei, 2017; Tao et al., 2019), while the low- and middle-income class (about 90\% of populations) obeys an exponential distribution (Nirei and Souma, 2007; Newby et al., 2011; Clementi et al., 2012; Prinz, 2016; Irwin and Irwin, 2017; Rosser, 2019; Tao et al., 2019; Ma and Ruzic, 2020). Theoretically, it has been known that the generalized Pareto distribution is a fairly general family that includes the Pareto distribution and the 
exponential distribution as two special cases (Balkema and Haan, 1974; Pickands, 1975; Singh and Maddala, 1976; Cowell, 2000). In this regard, Blanchet et al. (2017, 2018) used the generalized Pareto distribution to describe the income structure of the total population, where the generalized Pareto distribution is defined as follows:

$$
F_{\xi}(t \geq x)=\left(1+\xi \frac{x-\mu}{\theta}\right)^{-1 / \xi}
$$

where $\xi>0, x$ denotes the income level, and $F_{\xi}(t \geq x)$ denotes the fraction of population with the income being higher than $x$.

It can be seen that equation (1) yields the Pareto distribution $F_{\xi}(t \geq x)=$ $(x / \mu)^{-1 / \xi}$ when $\mu=\theta / \xi$ (Jenkins, 2016; Blanchet et al., 2017), where the Pareto exponent is denoted by $1 / \xi$, which measures the degree of income inequality, that is, a larger Pareto exponent is associated with lower income inequality (Jones and Kim, 2018). Intuitively, a lower income inequality should correspond to a more equalopportunity society. Thus, we anticipate that, as the Pareto exponent $1 / \xi \rightarrow \infty$ (or $\xi \rightarrow 0$ ), equation (1) yields a distribution being close to equal opportunity; that is,

$$
F_{0}(t \geq x)=\lim _{\xi \rightarrow 0}\left(1+\xi \frac{x-\mu}{\theta}\right)^{-1 / \xi}=e^{-\frac{x-\mu}{\theta}} .
$$

Equation (2) is an exponential distribution, which is expected to describe the income structure of an equal-opportunity economy. In the economic literature, a peer-to-peer economy without an intermediary third-party is recognized as an equal-opportunity market economy (Einav et al., 2016; Davidson et al., 2018; Filippi et al., 2020; Hojckova et al., 2020). In this regard, Tao $(2016,2021)$ has theoretically shown that the exponential income distribution emerges spontaneously in a peer-to-peer economic network that shares the publicly available technology $y^{2}$ :

$$
\left\{\begin{array}{l}
a_{j}=e^{-\frac{\varepsilon_{j}-\mu}{\theta}} \\
j=1, \ldots, n
\end{array},\right.
$$

where $\varepsilon_{1}<\varepsilon_{2}<\cdots<\varepsilon_{n}$, and $a_{j}$ denotes that there are $a_{j}$ households, each of which obtains $\varepsilon_{j}$ units of income. We will clarify the economic implication of $\mu$ and

\footnotetext{
${ }^{2}$ Equation (3) has been known as a Boltzmann-like distribution (Cerreia-Vioglio et al., 2020). The publicly available technology indicates that there is no monopoly on technology, which guarantees equal opportunity of market competition.
} 
$\theta$ in section 3 .

Because the exponential income distribution (3) is derived by assuming a peer-topeer economic network that shares the publicly available technology, we propose to identify the exponential income distribution as the benchmark structure of the wellfunctioning market economy. In this regard, Tao $(2016,2021)$ has proved that the exponential income distribution (3) is the spontaneous order of the peer-to-peer economic network that shares the publicly available technology: As a spontaneous order, the exponential income distribution (3) consists of a set of collective equilibrium decisions $^{3}$ among households (Tao, 2016, 2021). Next, we employ the empirical evidence to support this proposal.

Before doing this, we first demonstrate the relationship between equations (2) and (3). Equation (2) is the cumulative distribution of income, where the income level takes continuous value. By contrast, equation (3) is the density distribution of income, where the income level takes discrete values to keep up with reality. Tao et al (2019) have shown that the cumulative form of equation (3) is equivalent to equation (2) when the income level takes continuous value. From this sense, the discrete form (3) has included the continuous form (2) as a special example. In this paper, we will use the discrete form (3) of exponential income distribution to perform a theoretical analysis. By contrast, it is more convenient to carry out empirical investigation by using the continuous form (2).

Because there has been a large body of empirical literature to support that the exponential distribution (2) dominates an extremely large proportion of populations (Nirei and Souma, 2007; Newby et al., 2011; Clementi et al., 2012; Prinz, 2016; Irwin and Irwin, 2017; Rosser, 2019; Tao et al., 2019; Ma and Ruzic, 2020), here we simply use the household income data from two representative market-economy countries to support the validity of the exponential distribution (2) in describing the income structure of the well-functioning market economy. The two countries include one

\footnotetext{
3 Tao (2016) defines an economic order as a set of collective equilibrium decisions (or general equilibrium allocations) among $N$ agents. In this regard, the spontaneous order is defined as the likeliest set occurring (among all permissible sets).
} 
typical developed economy (the United Kingdom) and one typical developing economy (China). Figure 1 shows that the low- and middle-income classes (about 90\% of populations) in China and the United Kingdom uniformly obey the exponential distribution (2).

\section{[Insert Figure 1 here]}

In particular, because China is a special sample that has undergone the transition from a planned economy to a market economy, it is more important to examine if there is a transition towards an exponential income distribution after the market-oriented economic reformation. Figure 2 empirically shows that the transition of China's income structure evolving to an exponential income distribution occurred after the marketoriented economic reformation (i.e., 1978). The transition of China's income structure is compelling evidence for identifying the exponential income distribution as the benchmark structure of the well-functioning market economy.

\section{[Insert Figure 2 here]}

Because the empirical evidence has shown that the exponential distribution (2) dominates an extremely large proportion of populations, we assume that the income structure of the total population in a market economy is approximately described by an exponential distribution. Based on this assumption, we next use the discrete form (3) of exponential income distribution to verify that the technology factor in aggregate production function is exactly equal to society's information stock.

\section{Aggregate production function}

According to the economic meaning of exponential income distribution (3), the total number of households, $N$, and the GDP, $Y$, of the economy can be written as below:

$$
N=\sum_{j=1}^{n} a_{j}=\sum_{j=1}^{n} e^{-\frac{\varepsilon_{j}-\mu}{\theta}},
$$




$$
Y=\sum_{j=1}^{n} a_{j} \varepsilon_{j}=\sum_{j=1}^{n} \varepsilon_{j} e^{-\frac{\varepsilon_{j}-\mu}{\theta}} .
$$

Here, we order:

$$
\begin{aligned}
& \alpha=-\frac{\mu}{\theta}, \\
& \beta=\frac{1}{\theta} .
\end{aligned}
$$

By using equations (6) and (7), equations (4) and (5) can be rewritten as

$$
\begin{aligned}
& N=\sum_{j=1}^{n} \frac{1}{e^{\alpha+\beta \varepsilon_{j}}}, \\
& Y=\sum_{j=1}^{n} \frac{\varepsilon_{j}}{e^{\alpha+\beta \varepsilon_{j}} .}
\end{aligned}
$$

Equations (4) and (5) indicate that $N$ and $Y$ are related via two parameters $\alpha$ and $\beta$. This means that the aggregate production function (or GDP) $Y$ should be a function of total number of agents, $N$. By using equations (8) and (9), we can obtain

$$
d Y=-\frac{\alpha}{\beta} d N+\frac{1}{\beta} d\left(N-\alpha \frac{\partial N}{\partial \alpha}-\beta \frac{\partial N}{\partial \beta}\right) .
$$

The derivation for equation (10) can be found in Appendix A.

Let us order:

$$
T=N-\alpha \frac{\partial N}{\partial \alpha}-\beta \frac{\partial N}{\partial \beta} \text {. }
$$

The following proposition 1 guarantees that if equation (12) is solvable, then $N$ is independent of $T$ (Tao, 2019).

Proposition 1: Assume that $N$ agents obey the exponential income distribution (3) so that equation (10) holds. Then $N$ is independent of $T$ if and only if the following partial differential equation (12) holds and is solvable:

$$
N \frac{\partial Y(N, T)}{\partial N}+(T-N) \frac{\partial Y(N, T)}{\partial T}=Y(N, T)
$$

Proof. The proof can be found in Appendix B.

It is easy to verify that equation (12) is solvable (Tao, 2021). Henceforth, we always assume that equation (12) holds. Following this assumption, $N$ is independent of $T$. Therefore, in the context of exponential income distribution (3), we have the aggregate production function as below:

$$
Y=Y(N, T) .
$$


Substituting equations (6), (7), and (11) into equation (10) we obtain

$$
d Y(N, T)=\mu d N+\theta d T .
$$

Since $N$ is independent of $T$, by equation (14) we have

$$
\begin{aligned}
& \mu=\frac{\partial Y(N, T)}{\partial N}, \\
& \theta=\frac{\partial Y(N, T)}{\partial T} .
\end{aligned}
$$

To identify the economic implication of $\mu$ and $\theta$, we interpret $T$ as the technology factor. In section 4 , we will verify the validity of this interpretation. Here, we further assume that households are the owners of labor and capital. This assumption is natural. If a household has no labor or capital, it cannot survive in a real society. Therefore, the total number of households, $N$, can be regarded as the function of the labor $L$ and the capital stock $K$; that is, we have

$$
N=N(L, K) \text {. }
$$

In the context of neoclassical economics, the aggregate production function can be written as

$$
Y=Y(L, K, T) \text {. }
$$

Because equation (12) holds, $N$ is independent of $T$. Therefore, by equations (17) and (18), the aggregate production function (13) can be written in the form:

$$
Y=Y(N(L, K), T),
$$

which implies a Hicks-neutral-like technical progress.

By equations (15) and (17), $\mu$ denotes the marginal labor-capital return, which denotes the increment of GDP when a new household enters markets, while technology factor $T$ is held constant. Similarly, $\theta$ denotes the marginal technology return. The main purpose of this paper is to prove that if equation (12) holds, then the technology factor $T$ is exactly equal to society's information stock.

\section{Main results}

Let us first assume that the economy obeys the exponential income distribution (3). To prove that $T$ stands for society's information stock, we need to make three assumptions. 
Assumption 1: The GDP, $Y$, is a function of decentralized decisions among $N$ agents (or households $\left.{ }^{4}\right), \boldsymbol{s}=\left(s_{1}, s_{2}, \ldots, s_{N}\right)$; that is, $Y=Y(\boldsymbol{s})$, where $s_{i}$ denotes the economic decision of the $i$-th agent and $i=1, \ldots, N$.

Assumption 1 is derived from the presupposition of this paper that the economy obeys the exponential income distribution (3), which is the spontaneous order of the peer-to-peer economy that shares the publicly available technology (Tao, 2016, 2021). This order consists of a set of general equilibrium allocations among households (Tao, 2016, 2021), where each equilibrium allocation is a collection of decentralized equilibrium decisions among households, $\boldsymbol{s}=\left(s_{1}, s_{2}, \ldots, s_{N}\right)$. Thus, the economic meaning of Assumption 1 is that the GDP of a country is determined by the economic decision of each agent. This assumption is natural. Indeed, the GDP consists of all agents' incomes and an agent's income is determined by her economic decision. For example, a good decision may increase income and a bad decision may reduce income.

Furthermore, $\boldsymbol{s}=\left(s_{1}, s_{2}, \ldots, s_{N}\right)$ indicates that the number of agents is discrete (or countable). However, as with Aumann's research of a continuum of traders (Aumann, 1966), Assumption 1 can be applied to the case that the number of agents is continuous (or uncountable). For a continuum of agents, we order $s=\left(s_{i}\right)_{i \in R}$ with $R=[0, N]$.

Assumption 2: $N$ is not a function of $\boldsymbol{s}$. In particular, if the agent $i$ does not make economic decision (i.e., inactive), then we order $s_{i}=0$, where, for a discrete number of agents, we have $i=1, \ldots, N$, and, for a continuum of agents, we have $i \in R$.

Assumption 2 eliminates the possibility that $N$ is a function of $\boldsymbol{s}$. To understand this assumption, we simply consider the discrete case $\boldsymbol{s}=\left(s_{1}, s_{2}, \ldots, s_{N}\right)$. For example, if we $\operatorname{order}^{5} s_{3}=s_{4}=\cdots=s_{N}=0$, then we have $s=\left(s_{1}, s_{2}, 0 \ldots, 0\right)=\left(s_{1}, s_{2}\right)$, which has nothing to do with $N$. Furthermore, by the Assumption 2, we also have the following result: For $\boldsymbol{s}=\left(s_{1}, s_{2}, \ldots, s_{N}\right)$ and $\boldsymbol{s}^{\prime}=\left(s_{1}, s_{2}, \ldots, s_{N_{0}}\right)$, if $N_{0}<N$, then

\footnotetext{
${ }^{4}$ In this paper, we do not distinguish between agent and household.

${ }^{5} s_{3}=s_{4}=\cdots=s_{N}=0$ means that social members $i=1, \ldots, N$ are inactive.
} 
we may have $T(\boldsymbol{s})=T\left(\boldsymbol{s}^{\prime}\right)$. To see this, let us order $\boldsymbol{s}^{\prime \prime}=\left(s_{N_{0}+1}, \ldots, s_{N}\right)$. Then $\boldsymbol{s}=$ $\left(\boldsymbol{s}^{\prime}, \boldsymbol{s}^{\prime \prime}\right)$. In this case, if we consider $\boldsymbol{s}^{\prime \prime}=(0, \ldots, 0)$, then we have $\boldsymbol{s}=\left(\boldsymbol{s}^{\prime}, \boldsymbol{s}^{\prime \prime}\right)=$ $\left(\boldsymbol{s}^{\prime}, \mathbf{0}\right)=\boldsymbol{s}^{\prime}$; that is, $T(\boldsymbol{s})=T\left(\boldsymbol{s}^{\prime}\right)$. Obviously, these results can be applied to the case of a continuum of agents.

By Proposition 1, we have known that $Y$ is a function of $N$ and $T$; therefore, by Assumptions 1 and 2 we conclude that $T$ is a function of $\boldsymbol{s}$; that is,

$$
T=T(\boldsymbol{s}) \text {. }
$$

For the function $T=T(\boldsymbol{s})$, we further make the continuity assumption as below:

Assumption 3 (continuity): If there are two collective decentralized decisions $\boldsymbol{s}$ and $\boldsymbol{s}^{\prime}$ such that $T(\boldsymbol{s})<T\left(\boldsymbol{s}^{\prime}\right)$, then we do find a collective decentralized decision $\boldsymbol{s}^{\prime \prime}$ to satisfy $T(\boldsymbol{s})<T\left(\boldsymbol{s}^{\prime \prime}\right)<T\left(\boldsymbol{s}^{\prime}\right)$, where $\boldsymbol{s} \neq \boldsymbol{s}^{\prime} \neq \boldsymbol{s}^{\prime \prime}$.

Using equation (20) and Assumption 2, equation (14) can be rewritten as

$$
d Y(N, T(\boldsymbol{s}))=\mu d N+\theta d T(\boldsymbol{s}) .
$$

Based on Assumptions 1-3, we can prove three lemmas as below:

Lemma 1: Assume that $N$ agents obey the exponential income distribution (3) and that equation (12) holds. For any given number $T_{0}$, if the probability of each agent acquiring income is independent, the probability of $N$ agents taking the decentralized decisions $\boldsymbol{s}$ is denoted by

$$
P(\boldsymbol{s})=\frac{1}{Z} e^{-\frac{Y(s)-N \cdot \mu}{\theta}}
$$

with

$$
Z=\sum_{\boldsymbol{s}^{\prime} \in\left\{\boldsymbol{r}^{\prime} \mid T\left(\boldsymbol{r}^{\prime}\right)=T_{0}\right\}} e^{-\frac{Y\left(\boldsymbol{s}^{\prime}\right)-N \cdot \mu}{\theta}}
$$

where $\boldsymbol{s} \in\left\{\boldsymbol{r} \mid T(\boldsymbol{r})=T_{0}\right\}, Y(\boldsymbol{s})=Y(N, T(\boldsymbol{s}))$, and the summation $\left.\sum_{\boldsymbol{s}^{\prime} \in\left\{\boldsymbol{r}^{\prime} \mid T\left(\boldsymbol{r}^{\prime}\right)=T_{0}\right.}\right\}^{*}$ in equation (23) runs over all decentralized decisions $\boldsymbol{s}^{\prime}$ in the set $\left\{\boldsymbol{r}^{\prime} \mid T\left(\boldsymbol{r}^{\prime}\right)=T_{0}\right\}$.

Proof. The proof can be found in Appendix C.

Using equations (22) and (23), it is easy to verify 


$$
\sum_{\boldsymbol{s} \in\left\{\boldsymbol{r} \mid T(\boldsymbol{r})=T_{0}\right\}} P(\boldsymbol{s})=1 .
$$

Lemma 2: Assume that $Y(N, T(\boldsymbol{s}))$ is a differentiable function with respect to $N$ and $T(\boldsymbol{s})$. For a continuum of agents, if equation (21) holds, then we have

$$
d \bar{Y}=\mu d N+\theta d \bar{T}
$$

where

$$
\begin{aligned}
\bar{Y} & =\sum_{\boldsymbol{s} \in\left\{\boldsymbol{r} \mid T(\boldsymbol{r})=T_{0}\right\}} Y(N, T(\boldsymbol{s})) \cdot P(\boldsymbol{s}), \\
\bar{T} & =\sum_{\boldsymbol{s} \in\left\{\boldsymbol{r} \mid T(\boldsymbol{r})=T_{0}\right\}} T(\boldsymbol{s}) \cdot P(\boldsymbol{s}),
\end{aligned}
$$

with $T_{0}$ being any given number.

Proof. The proof can be found in Appendix D.

Substituting equations (6) and (7) into equations (22) and (23) yields

$$
\begin{aligned}
& P(\boldsymbol{s})=\frac{1}{Z} e^{-\beta Y(\boldsymbol{s})-\alpha N}, \\
& Z=\sum_{\boldsymbol{s}^{\prime} \in\left\{\boldsymbol{r}^{\prime} \mid T\left(\boldsymbol{r}^{\prime}\right)=T_{0}\right\}} e^{-\beta Y\left(\boldsymbol{s}^{\prime}\right)-\alpha N} .
\end{aligned}
$$

Lemma 3: For a given constant $T_{0}$, if equation (11) holds, by using equations (28) and (29) one has

$$
\begin{aligned}
& N=-\frac{\partial}{\partial \alpha} \ln Z, \\
& \bar{Y}=-\frac{\partial}{\partial \beta} \ln Z,
\end{aligned}
$$

where $\bar{Y}=\sum_{\boldsymbol{s} \in\left\{\boldsymbol{r} \mid T(\boldsymbol{r})=T_{0}\right\}} Y(N, T(\boldsymbol{s})) \cdot P(\boldsymbol{s})$ and $Z=\sum_{\boldsymbol{s} \in\left\{\boldsymbol{r} \mid T(\boldsymbol{r})=T_{0}\right\}} e^{-\beta Y(\boldsymbol{s})-\alpha N}$.

Proof. The proof can be found in Appendix E.

Using Lemmas 1-3, we start to prove the central proposition of this paper.

Proposition 2: For any given number $T_{0}$, if $N$ agents obey the exponential income distribution (3) and if equation (12) holds, then one has

$$
T(\boldsymbol{s})=-\ln P(\boldsymbol{s}),
$$

where $\boldsymbol{s} \in\left\{\boldsymbol{r} \mid T(\boldsymbol{r})=T_{0}\right\}$. 
Proof. It is easy to verify the following two equations:

$$
\begin{aligned}
& d\left(\alpha \frac{\partial}{\partial \alpha} \ln Z\right)=\frac{\partial}{\partial \alpha} \ln Z d \alpha+\alpha d\left(\frac{\partial}{\partial \alpha} \ln Z\right), \\
& d\left(\beta \frac{\partial}{\partial \beta} \ln Z\right)=\frac{\partial}{\partial \beta} \ln Z d \beta+\beta d\left(\frac{\partial}{\partial \beta} \ln Z\right)
\end{aligned}
$$

Substituting equations (30) and (31) into equations (33) and (34) we can obtain

$$
d\left(\alpha \frac{\partial}{\partial \alpha} \ln Z+\beta \frac{\partial}{\partial \beta} \ln Z\right)=d \ln Z+\beta d \bar{Y}+\alpha d N,
$$

where we have used $\operatorname{dn} Z=\frac{\partial}{\partial \alpha} \ln Z \cdot d \alpha+\frac{\partial}{\partial \beta} \ln Z \cdot d \beta$.

Equation (35) can be rewritten as

$$
d \bar{Y}=-\frac{\alpha}{\beta} d N+\frac{1}{\beta} d\left(\ln Z-\alpha \frac{\partial}{\partial \alpha} \ln Z-\beta \frac{\partial}{\partial \beta} \ln Z\right),
$$

Because equation (12) holds, by Proposition $1, N$ is independent of $T$. Therefore, equation (21) holds. By using Lemma 2 and equation (21), equation (25) holds.

Comparing equations (25) and (36), by using equations (6) and (7) we have

$$
\bar{T}=\ln Z-\alpha \frac{\partial}{\partial \alpha} \ln Z-\beta \frac{\partial}{\partial \beta} \ln Z .
$$

Substituting equation (29) into equation (37) one has

$$
\bar{T}=-\sum_{\boldsymbol{s} \in\left\{\boldsymbol{r} \mid T(\boldsymbol{r})=T_{0}\right\}} P(\boldsymbol{s}) \cdot \ln P(\boldsymbol{s}),
$$

where we have used equation (28).

Furthermore, substituting equation (12) into equation (22) yields

$$
P(\boldsymbol{s})=\frac{e^{-T(\boldsymbol{s})}}{\sum_{\boldsymbol{s}^{\prime} \in\left\{\boldsymbol{r}^{\prime} \mid T\left(\boldsymbol{r}^{\prime}\right)=T_{0}\right\}} e^{-T\left(\boldsymbol{s}^{\prime}\right)}},
$$

where we have used equations (15) and (16).

Combining equations (27) and (38) we have

$$
\sum_{\boldsymbol{s} \in\left\{\boldsymbol{r} \mid T(\boldsymbol{r})=T_{0}\right\}} T(\boldsymbol{s}) P(\boldsymbol{s})=-\sum_{\boldsymbol{s} \in\left\{\boldsymbol{r} \mid T(\boldsymbol{r})=T_{0}\right\}} P(\boldsymbol{s}) \ln P(\boldsymbol{s}) .
$$

Substituting equation (39) into equation (40) we have

$$
\sum_{\boldsymbol{s} \in\left\{\boldsymbol{r} \mid T(\boldsymbol{r})=T_{0}\right\}} e^{-T(\boldsymbol{s})}=1,
$$

which implies that equation (39) can be simplified to

$$
P(\boldsymbol{s})=e^{-T(\boldsymbol{s})}
$$

that is,

$$
T(\boldsymbol{s})=-\ln P(\boldsymbol{s}) .
$$


Equation (32) is the main result of this paper. By equations (24) and (32), we can provide an economic interpretation for the technology factor $T(\boldsymbol{s})$. According to Shannon's information theory (Shannon, 1948), if the probability of an event $A$ occurring is denoted by $P(A)$, then the information content contained in the event $A$ is equal to $-\ln P(A)$. This means that the lower the probability of an event $A$ occurring, the larger the information content contained in the event $A$. By Lemma 1, we have acknowledged that $P(\boldsymbol{s})$ denotes the probability of $N$ agents taking decentralized decisions $\boldsymbol{s}$. Therefore, by equation (32), the technology factor $T(\boldsymbol{s})$ in the aggregate production function (19) denotes the information content contained in the event of $N$ agents taking decentralized decisions $\boldsymbol{s}$. Because each agent $i$ makes decision $s_{i}$ based on personal information or dispersed knowledge (Hayek, 1945) and because decentralized decisions $\boldsymbol{s}=\left(s_{1}, s_{2}, \ldots, s_{N}\right)$ cover all agents in the society, taking decentralized decisions $\boldsymbol{s}$ can be regarded as a process of integrating all of personal information. Therefore, we denote $T(\boldsymbol{s})=-\ln P(\boldsymbol{s})$ by this society's information stock. From this sense, the technological progress is a process of integrating all of decentralized decisions. In this regard, Hayek (1988) has inferred that enhancing productivity is a procedure for combining different and scattered information in markets. This suggests that our result (32) actually formalizes his inference.

However, our result does not mean that decentralized decisions always lead to technological progress. In our setting, the economy is supposed to be a peer-to-peer economic network that shares the publicly available technology such that the exponential income distribution (3) emerges spontaneously. In particular, the exponential income distribution (3) consists of a set of general equilibrium allocations among households (Tao, 2016, 2021), in each of which all households reach consensuses on resource allocation. This suggests that decentralized decisions cause technological progress when all households reach consensuses on these decisions. Furthermore, by equation (32), the phenomenon of a society evolving to a high technological level should be a low probability event of collective decisions. This result agrees with our observation. To our knowledge, among all the species on the earth, only 
humans evolved to a high technological civilization.

\section{Conclusion}

In this paper, we argue that exponential income distribution is a signature of the wellfunctioning market economy. Empirically, we use the household income data from two representative market-economy countries to support the validity of the exponential income distribution in describing the income structure of the well-functioning market economy. The two countries include one typical developed economy (the United Kingdom) and one typical developing economy (China). In particular, because China is a special sample that has undergone the transition from a planned economy to a market economy, we carefully examine whether there is a transition towards an exponential income distribution after the market-oriented economic reformation. The empirical investigation has verified that the transition of China's income structure evolving to an exponential income distribution occurred after the market-oriented economic reformation (i.e., 1978). The transition of China's income structure is compelling evidence for identifying the exponential income distribution as a signature of the well-functioning market economy. Based on the empirical investigation, we assume that the income structure of the total population in a market economy is approximately described by an exponential distribution. Thus, we strictly prove that, if the income structure of an economy obeys an exponential distribution, the income summation over all households leads to a neoclassical aggregate production function, in which the technology factor is exactly equal to society's information stock. This theoretical finding formalizes the validity of interpreting technology factor in the theory of economic growth as "knowledge" or "information stock". 


\section{Appendix A}

By equations (8) and (9), one has

$$
\begin{gathered}
\frac{\partial N}{\partial \alpha}=-N, \\
\frac{\partial N}{\partial \beta}=-Y .
\end{gathered}
$$

The differential of equation (A.2) yields

$$
d Y=-d\left(\frac{\partial N}{\partial \beta}\right)=-\frac{1}{\beta} d\left(\beta \frac{\partial N}{\partial \beta}\right)+\frac{1}{\beta} \frac{\partial N}{\partial \beta} d \beta .
$$

By equation (8), $N$ is a function of $\alpha$ and $\beta$; therefore, the complete differential of $N$ is

$$
d N=\frac{\partial N}{\partial \alpha} d \alpha+\frac{\partial N}{\partial \beta} d \beta,
$$

which leads to

$$
\frac{\partial N}{\partial \beta} d \beta=d N-\frac{\partial N}{\partial \alpha} d \alpha .
$$

Substituting equation (A.5) into equation (A.3) yields

$$
d Y=-\frac{1}{\beta} d\left(\beta \frac{\partial N}{\partial \beta}\right)+\frac{1}{\beta} d N-\frac{1}{\beta} \frac{\partial N}{\partial \alpha} d \alpha .
$$

On the other hand, we have

$$
d\left(\alpha \frac{\partial N}{\partial \alpha}\right)=\alpha d\left(\frac{\partial N}{\partial \alpha}\right)+\frac{\partial N}{\partial \alpha} d \alpha,
$$

which leads to

$$
\frac{\partial N}{\partial \alpha} d \alpha=d\left(\alpha \frac{\partial N}{\partial \alpha}\right)-\alpha d\left(\frac{\partial N}{\partial \alpha}\right) .
$$

Substituting equation (A.8) into equation (A.6) yields

$$
\begin{aligned}
d Y & =-\frac{1}{\beta} d\left(\beta \frac{\partial N}{\partial \beta}\right)+\frac{1}{\beta} d N-\frac{1}{\beta} d\left(\alpha \frac{\partial N}{\partial \alpha}\right)+\frac{\alpha}{\beta} d\left(\frac{\partial N}{\partial \alpha}\right) \\
& =\frac{\alpha}{\beta} d\left(\frac{\partial N}{\partial \alpha}\right)+\frac{1}{\beta} d\left(N-\alpha \frac{\partial N}{\partial \alpha}-\beta \frac{\partial N}{\partial \beta}\right) .
\end{aligned}
$$

By equation (A.1), one can rewrite equation (A.9) in the form:

$$
d Y=-\frac{\alpha}{\beta} d N+\frac{1}{\beta} d\left(N-\alpha \frac{\partial N}{\partial \alpha}-\beta \frac{\partial N}{\partial \beta}\right) .
$$




\section{Appendix B}

\section{Proof of Proposition 1}

Proof. We first verify the sufficiency.

Substituting equations (A.1) and (A.2) into equation (11) we have

$T=N+\alpha N+\beta Y$.

Because $T$ is independent of $N$, by equation (10), we get

$$
\begin{aligned}
& \frac{\partial Y}{\partial N}=-\frac{\alpha}{\beta}, \\
& \frac{\partial Y}{\partial T}=\frac{1}{\beta} .
\end{aligned}
$$

Substituting equations (B.2) and (B.3) into equation (B.1) yields equation (12).

Next, we verify the necessity. If equation (12) holds and is solvable, then $N$ is obviously independent of $T$. Furthermore, Tao (2019) has proved that equation (12) is solvable.

\section{Appendix C}

\section{Proof of Lemma 1}

Proof. If the income structure of an $\mathrm{N}$-agent society obeys the exponential income distribution (3), the joint probability distribution among $N$ agents, $P^{*}(1, \ldots, N)$, can be written as

$$
P^{*}(1, \ldots, N)=\prod_{j=1}^{n}\left(a_{j} / N\right)^{a_{j}} .
$$

To obtain equation (C.1), we first assume that the probability of each agent acquiring income is independent. By equation (4), the probability of obtaining $\varepsilon_{j}$ units of income is $a_{j} / N$. Therefore, by the assumption of independence, the joint probability among $a_{j}$ agents, each of which obtains $\varepsilon_{j}$ units of income, is $\left(a_{j} / N\right)^{a_{j}}=$ $\overbrace{\left(a_{j} / N\right) \cdot\left(a_{j} / N\right) \cdots\left(a_{j} / N\right)}^{a_{j}}$. Equation (C.1) is the result of considering all income levels $\varepsilon_{1}<\varepsilon_{2}<\cdots<\varepsilon_{n}$.

Substituting equation (3) into equation (C.1) yields 


$$
P^{*}(1, \ldots, N)=\frac{\prod_{j=1}^{n} N^{-a_{j}}}{\prod_{j=1}^{n} e^{\frac{a_{j} \varepsilon_{j}-a_{j} \mu}{\theta}}}=\frac{N^{-\sum_{j=1}^{n} a_{j}}}{e^{\frac{\sum_{j=1}^{n} a_{j} \varepsilon_{j}-\left(\sum_{j=1}^{n} a_{j}\right) \mu}{\theta}}} .
$$

Finally, we plug equations (4) and (5) into equation (C.2) to obtain

$$
P^{*}(1, \ldots, N)=\frac{N^{-N}}{e^{\frac{Y-N \cdot \mu}{\theta}}}
$$

By Assumption 1, $Y$ is a function of $\boldsymbol{s}$; that is,

$$
Y=Y(\boldsymbol{s}) \text {. }
$$

Using equation (C.4), $P^{*}(1, \ldots, N)$ can be written in the form of normalized probability:

$$
P(\boldsymbol{s})=\frac{P^{*}(1, \ldots, N)}{\sum_{\boldsymbol{s} \in\left\{r \mid T(r)=T_{0}\right\}} P^{*}(1, \ldots, N)},
$$

which by using equation (C.3) can be written as

$$
P(\boldsymbol{s})=\frac{1}{Z} e^{-\frac{Y(s)-N \cdot \mu}{\theta}},
$$

where

$$
Z=\sum_{\boldsymbol{s} \in\left\{\boldsymbol{r} \mid T(\boldsymbol{r})=T_{0}\right\}} e^{-\frac{Y(\boldsymbol{s})-N \cdot \mu}{\theta}} .
$$

and the summation $\sum_{\boldsymbol{s} \in\left\{\boldsymbol{r} \mid T(\boldsymbol{r})=T_{0}\right\}}$ runs over all possible decentralized decisions $\boldsymbol{S}$ that satisfy $T(\boldsymbol{s})=T_{0}$.

\section{Appendix D}

\section{Proof of Lemma 2}

Proof. We consider any two collections of decentralized decisions $\boldsymbol{s}$ and $\boldsymbol{s}^{\prime}$ and two numbers $N$ and $N^{\prime}$, respectively; that is, $N$ agents take the decentralized decision $\boldsymbol{s}$ and $N^{\prime}$ agents take the decentralized decision $\boldsymbol{s}^{\prime}$. Then we order

$$
\begin{gathered}
\Delta N=N^{\prime}-N, \\
\Delta T=T\left(\boldsymbol{s}^{\prime}\right)-T(\boldsymbol{s}),
\end{gathered}
$$

where $\Delta N \neq 0$ and $\Delta T \neq 0$. Therefore, we have $\boldsymbol{s}^{\prime} \neq \boldsymbol{s}$.

Because $Y(N, T(s))$ is a differentiable function with respect to $N$ and $T(\boldsymbol{s})$, by using the mean value theorem of differential, $Y\left(N^{\prime}, T\left(s^{\prime}\right)\right)$ can be written as

$$
Y\left(N^{\prime}, T\left(\boldsymbol{s}^{\prime}\right)\right)
$$




$$
=Y(N, T(\boldsymbol{s}))+\frac{\partial}{\partial N} Y\left(N+\eta \Delta N, T\left(\boldsymbol{s}^{\prime \prime}\right)\right) \Delta N+\frac{\partial}{\partial T} Y\left(N+\eta \Delta N, T\left(\boldsymbol{s}^{\prime \prime}\right)\right) \Delta T,
$$

where $0<\eta<1$ and, by Assumption 3, the decentralized decision $\boldsymbol{s}^{\prime \prime}$ satisfies $T(\boldsymbol{s})<T\left(\boldsymbol{s}^{\prime \prime}\right)<T\left(\boldsymbol{s}^{\prime}\right)$.

Equation (D.3) can be rewritten as

$$
\begin{aligned}
& Y\left(N^{\prime}, T\left(\boldsymbol{s}^{\prime}\right)\right)-Y(N, T(\boldsymbol{s})) \\
& =\frac{\partial}{\partial N} Y\left(N+\eta \Delta N, T\left(\boldsymbol{s}^{\prime \prime}\right)\right) \Delta N+\frac{\partial}{\partial T} Y\left(N+\eta \Delta N, T\left(\boldsymbol{s}^{\prime \prime}\right)\right) \Delta T .
\end{aligned}
$$

Let us order $T\left(\boldsymbol{s}^{\prime}\right)=T_{0}^{\prime}$ and $T(\boldsymbol{s})=T_{0}$ with $T_{0}^{\prime}$ and $T_{0}$ being two constants. Because $T(\boldsymbol{s})<T\left(\boldsymbol{s}^{\prime \prime}\right)<T\left(\boldsymbol{s}^{\prime}\right)$, we conclude $\boldsymbol{s} \neq \boldsymbol{s}^{\prime \prime} \neq \boldsymbol{s}^{\prime}$. Therefore, by acting the summation operator $\sum_{\boldsymbol{s} \in\left\{\boldsymbol{r} \mid T(\boldsymbol{r})=T_{0}\right\}} \sum_{\boldsymbol{s}^{\prime} \in\left\{\boldsymbol{r}^{\prime} \mid T\left(\boldsymbol{r}^{\prime}\right)=T_{0}^{\prime}\right\}} P(\boldsymbol{s}) P\left(\boldsymbol{s}^{\prime}\right)$ on both sides of equation (D.4) we have

$$
\bar{Y}^{\prime}-\bar{Y}=\frac{\partial}{\partial N} Y\left(N+\eta \Delta N, T\left(\boldsymbol{s}^{\prime \prime}\right)\right) \Delta N+\frac{\partial}{\partial T} Y\left(N+\eta \Delta N, T\left(\boldsymbol{s}^{\prime \prime}\right)\right) \Delta \bar{T},
$$

where

$$
\begin{aligned}
& \bar{Y}^{\prime}=\sum_{\boldsymbol{s}^{\prime} \in\left\{\boldsymbol{r}^{\prime} \mid T\left(\boldsymbol{r}^{\prime}\right)=T_{0}^{\prime}\right\}} Y\left(N^{\prime}, T\left(\boldsymbol{s}^{\prime}\right)\right) P\left(\boldsymbol{s}^{\prime}\right) \\
& \bar{Y}=\sum_{\boldsymbol{s} \in\left\{\boldsymbol{r} \mid T(\boldsymbol{r})=T_{0}\right\}} Y(N, T(\boldsymbol{s})) P(\boldsymbol{s}) \\
& \Delta \bar{T}=\sum_{\boldsymbol{s}^{\prime} \in\left\{\boldsymbol{r}^{\prime} \mid T\left(\boldsymbol{r}^{\prime}\right)=T_{0}^{\prime}\right\}} T\left(\boldsymbol{s}^{\prime}\right) P\left(\boldsymbol{s}^{\prime}\right)-\sum_{\boldsymbol{s} \in\left\{\boldsymbol{r} \mid T(\boldsymbol{r})=T_{0}\right\}} T(\boldsymbol{s}) P(\boldsymbol{s}) .
\end{aligned}
$$

To derive equation (D.5), we have used $\sum_{\boldsymbol{s} \in\left\{\boldsymbol{r} \mid T(\boldsymbol{r})=T_{0}\right\}} P(\boldsymbol{s})=1$ and $\sum_{\boldsymbol{s}^{\prime} \in\left\{\boldsymbol{r}^{\prime} \mid T\left(\boldsymbol{r}^{\prime}\right)=T_{0}^{\prime}\right\}} P\left(\boldsymbol{s}^{\prime}\right)=1$.

Performing the limit operator on both sides of equation (D.5) as below:

$$
\begin{aligned}
& \operatorname{Lim}_{\Delta N \rightarrow 0} \lim _{\Delta T \rightarrow 0}\left[\bar{Y}^{\prime}-\bar{Y}\right]=\lim _{\Delta N \rightarrow 0} \lim _{\Delta T \rightarrow 0}\left[\frac{\partial}{\partial N} Y\left(N+\eta \Delta N, T\left(\boldsymbol{s}^{\prime \prime}\right)\right) \Delta N\right]+ \\
& \lim _{\Delta N \rightarrow 0} \lim _{\Delta T \rightarrow 0}\left[\frac{\partial}{\partial T} Y\left(N+\eta \Delta N, T\left(\boldsymbol{s}^{\prime \prime}\right)\right) \Delta \bar{T}\right] .
\end{aligned}
$$

Up to the first-order infinitesimal terms, equation (D.9) can be written as

$$
d \bar{Y}=\frac{\partial}{\partial N} Y(N, T) d N+\frac{\partial}{\partial T} Y(N, T) d \bar{T},
$$

which by equations (15) and (16) yields

$$
d \bar{Y}=\mu d N+\theta d \bar{T} .
$$




\section{Appendix E}

\section{Proof of Lemma 3}

Proof. Substituting equations (A.1) and (A.2) into equation (11) yields

$$
T=N+\alpha N+\beta Y \text {. }
$$

Let us order $T=T_{0}$. By equation (E.1) we have

$$
\begin{aligned}
& \frac{\partial T_{0}}{\partial \alpha}=\frac{\partial N}{\partial \alpha}+N+\alpha \frac{\partial N}{\partial \alpha}+\beta \frac{\partial Y}{\partial \alpha}=0, \\
& \frac{\partial T_{0}}{\partial \beta}=\frac{\partial N}{\partial \beta}+\alpha \frac{\partial N}{\partial \beta}+Y+\beta \frac{\partial Y}{\partial \beta}=0 .
\end{aligned}
$$

By using equations (A.1) and (A.2), equations (E.2) and (E.3) can be rewritten as

$$
\begin{aligned}
& \alpha \frac{\partial N}{\partial \alpha}+\beta \frac{\partial Y}{\partial \alpha}=0 \\
& \alpha \frac{\partial N}{\partial \beta}+\beta \frac{\partial Y}{\partial \beta}=0 .
\end{aligned}
$$

By using equation (29) we have

$$
\frac{\partial}{\partial \alpha} \ln Z=\frac{\sum_{s \in\left\{r \mid T(r)=T_{0}\right\}} e^{-\beta Y(s)-\alpha N}\left[-\beta \frac{\partial Y(s)}{\partial \alpha}-N-\alpha \frac{\partial N}{\partial \alpha}\right]}{\sum_{s^{\prime} \in\left\{\boldsymbol{r}^{\prime} \mid T\left(\boldsymbol{r}^{\prime}\right)=T_{0}\right\}} e^{-\beta Y\left(\boldsymbol{s}^{\prime}\right)-\alpha N}},
$$

which, by using equation (E.4), yields

$$
\frac{\partial}{\partial \alpha} \ln Z=\frac{-\sum_{\boldsymbol{s} \in\left\{\boldsymbol{r} \mid T(\boldsymbol{r})=T_{0}\right\}} N e^{-\beta Y(\boldsymbol{s})-\alpha N}}{\sum_{\boldsymbol{s}^{\prime} \in\left\{\boldsymbol{r}^{\prime} \mid T\left(\boldsymbol{r}^{\prime}\right)=T_{0}\right\}} e^{-\beta Y\left(\boldsymbol{s}^{\prime}\right)-\alpha N}}=-\sum_{\boldsymbol{s} \in\left\{\boldsymbol{r} \mid T(\boldsymbol{r})=T_{0}\right\}} N P(\boldsymbol{s})=-N .
$$

Similarly, by using equation (29) we have

$$
\frac{\partial}{\partial \beta} \ln Z=\frac{\sum_{s \in\left\{r \mid T(r)=T_{0}\right\}} e^{-\beta Y(s)-\alpha N}\left[-Y(s)-\beta \frac{\partial Y(s)}{\partial \beta}-\alpha \frac{\partial N}{\partial \beta}\right]}{\sum_{s^{\prime} \in\left\{r^{\prime} \mid T\left(r^{\prime}\right)=T_{0}\right\}} e^{-\beta Y\left(s^{\prime}\right)-\alpha N}},
$$

which, by using equation (E.5), yields

$$
\frac{\partial}{\partial \beta} \ln Z=\frac{-\sum_{\boldsymbol{s} \in\left\{\boldsymbol{r} \mid T(\boldsymbol{r})=T_{0}\right\}} Y(\boldsymbol{s}) e^{-\beta Y(\boldsymbol{s})-\alpha N}}{\sum_{\boldsymbol{s}^{\prime} \in\left\{\boldsymbol{r}^{\prime} \mid T\left(\boldsymbol{r}^{\prime}\right)=T_{0}\right\}} e^{-\beta Y\left(\boldsymbol{s}^{\prime}\right)-\alpha N}}=-\sum_{\boldsymbol{s} \in\left\{\boldsymbol{s} \mid T(\boldsymbol{s})=T_{0}\right\}} Y(\boldsymbol{s}) P(\boldsymbol{s})=-\bar{Y}
$$

To derive equations (E.7) and (E.9), we have used equation (28). 


\section{References:}

Acemoglu, D., Akcigit, U., and Kerr, W. R. (2016), "Innovation network". Proceedings of the National Academy of Sciences of the United States of America 113, $11483-11488$

Aoki, S. and Nirei, M. (2017), “Zipf's Law, Pareto’s Law, and the Evolution of Top Incomes in the United States". American Economic Journal: Macroeconomics 9, 3671.

Arrow, K. J. (1962), “The Economic Implications of Learning by Doing”. Review of Economic Studies 29, 155-173

Arthur, B. (2018), The Nature of Technology: What It Is and How It Evolves. Free Press.

Atkinson, A. B., Piketty, T. and Saez, E. (2011), “Top Incomes in the Long Run of History". Journal of Economic Literature 49, 3-71

Aumann, R. (1966), "Existence of competitive equilibria in markets with a continuum of traders". Econometrica 34, 1-17

Barro, R. J. (1990), “Government Spending in a Simple Model of Endogeneous Growth". Journal of Political Economy 98, S103-S125

Blanchet, T., Fournier, J. and Piketty, T. (2017), "Generalized Pareto Curves: Theory and Applications". Working Papers 201703, World Inequality Lab.

Blanchet, T., Garbinti, B., Goupille-Lebret, J. and Martínez-Toledano. C. (2018), "Applying Generalized Pareto Curves to Inequality Analysis". AEA Papers and Proceedings 108, 114-118

Cerreia-Vioglio, S., Maccheroni, F., Marinacci, M., Rustichini, A. (2020), “Axiomatic Tests for the Boltzmann Distribution”. arXiv: 2006.15970

Clementi, F., Gallegati, M. and Kaniadakis, G. (2012), “A new model of income distribution: the $\kappa$-generalized distribution". Journal of Economics 105, 63-91.

Cowell, F. A. (2000), Measuring Inequality. LSE Economic Series. Oxford University Press.

Davidson, S., Filippi, P. D., and Potts, J. (2018), "Blockchains and the economic 
institutions of capitalism". Journal of Institutional Economics 14, $639-658$

Einav, L., Farronato, C., and Levin, J. (2016), "Peer-to-Peer Markets". Annual Review of Economics 8, 615-635

Filippi, P. D., Mannan, M., Reijers, W. (2020), "Blockchain as a confidence machine: The problem of trust \& challenges of governance". Technology in Society 62 , 101284

Hayek, F. A. (1945), "The Use of Knowledge in Society". American Economic Review 35, 519-530

Hayek, F. A. (1988), The Fatal Conceit. Routledge

Hojckova, K., Ahlborg, H., Morrison, G. M., Sanden, B. (2020), “Entrepreneurial use of context for technological system creation and expansion: The case of blockchainbased peer-to-peer electricity trading”. Research Policy 49, 104046

Irwin. R. J. and Irwin, T. C. (2017), "Stability of an exponential distribution for New Zealand taxable personal income”. New Zealand Economic Papers 51, 49-59

Jenkins, S. P. (2016), "Pareto Models, Top Incomes and Recent Trends in UK Income Inequality”. Economica 84, 261-289

Jones, C. I. (1995), "R \& D-Based Models of Economic Growth”. Journal of Political Economy 103, 759-784

Jones, C. I. and Kim, J. (2018), “A Schumpeterian Model of Top Income Inequality”. Journal of Political Economy 126, 1785-1826

Lin, J. Y. (2011), "New Structural Economics: A Framework for Rethinking Development". The World Bank Research Observer 26, 193-221

Lucas, R. E. (1988), “On the Mechanics of Economic Development". Journal of Monetary Economics 22, 3-42

Mandelbrot, B. (1960), "The Pareto-Levy law and the distribution of income". International Economic Review 1, 79-106.

Mas-Colell, A., Whinston, M. D. and Green, J. R. (1995), Microeconomic Theory. Oxford University Press.

Ma, L. and Ruzic, D. (2020), "Globalization and top income shares." Journal of International Economics 125, 103312 
Murrell, P. (1992), "Evolutionary and Radical Approaches to Economic Reform". Economics of Planning 25, 79-95

Newby, M., Behr, A., and Feizabadi, M. S. (2011), "Investigating the distribution of personal income obtained from the recent U.S. data. Economic Modelling 28, 11701173

Nirei, M. and Souma, W. (2007), “A Two Factor Model of Income Distribution Dynamics". Review of Income and Wealth 53, 440-459

Nordhaus, W. (2021), “Are We Approaching an Economic Singularity? Information Technology and the Future of Economic Growth". American Economic Journal: Macroeconomics 13, 299-332

Prinz, A. (2016), “Do capitalistic institutions breed billionaires?”. Empirical Economics 51, 1319-1332

Romer, D. (2012), Advanced Macroeconomics (Fourth Edition), The McGraw-Hill Companies, Inc.

Romer, P. M. (1986), “Increasing Returns and Long-Run Growth”. Journal of Political Economy 94, 1002-1037.

Romer, P. M. (1990), “Endogenous Technological Change”. Journal of Political Economy 98, S71-S102

Rosser, J. B. (2019), "The Minsky moment as the revenge of entropy." Macroeconomic Dynamics 24, 7-23

Saviotti, P. P. (1988), "Information, variety and entropy in technoeconomic development". Research Policy 17, 89-103

Shannon, C. E. (1948), “A Mathematical Theory of Communication”. Bell System Technical Journal 27, 379-423

Singh, S. K. and Maddala, G. S. (1976), "A function for the size distribution of income". Econometrica 44, 963-970

Solow, R. M. (1956), "A Contribution to the Theory of Economic Growth". Quarterly Journal of Economics 70, 65-94

Tao, Y. (2016), “Spontaneous economic order”. Journal of Evolutionary Economics 26, $467-500$ 
Tao, Y., Wu, X., Zhou, T., Yan, W., Huang, Y., Yu, H., Mondal, B., and Yakovenko, V. M. (2019), "Exponential structure of income inequality: evidence from 67 countries". Journal of Economic Interaction and Coordination 14, 345-376.

Tao, Y. (2021), "Exponential Income Distribution and Evolution of Unemployment Compensation in the United Kingdom". Preprints 2021, 2021070350 (doi: 10.20944/preprints202107.0350.v1). 


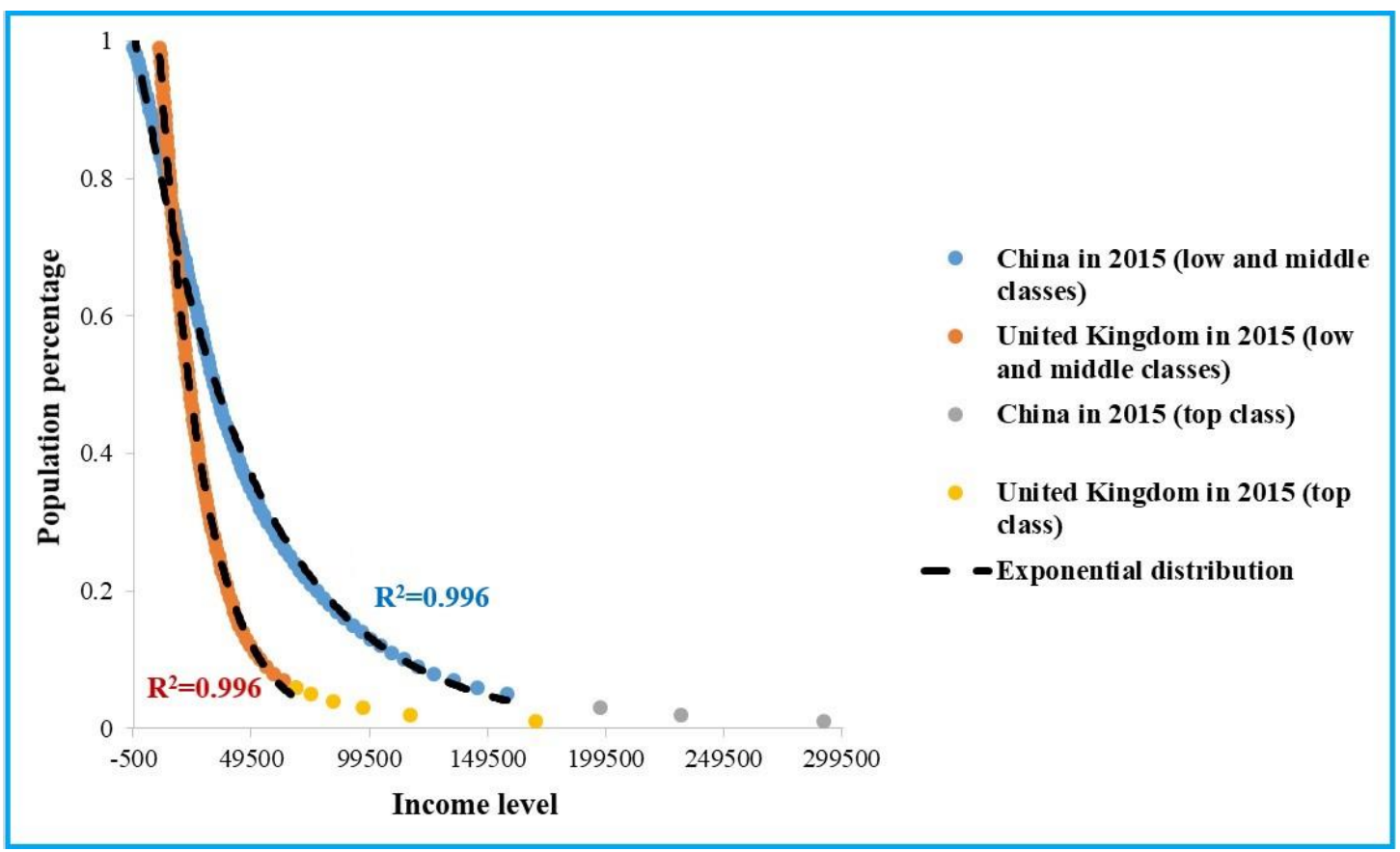

Figure 1: The low- and middle-income classes (about $90 \%$ of populations) in $\mathrm{China}^{6}$ and the United Kingdom ${ }^{7}$ uniformly obey the exponential income distribution.

${ }^{6}$ Data resource for China: $\mathrm{http}: / /$ wid.world/data/

7 Data resource for the United Kingdom: https://www.gov.uk/government/statistics/percentilepoints-from-1-to-99-for-total-income-before-and-after-tax 


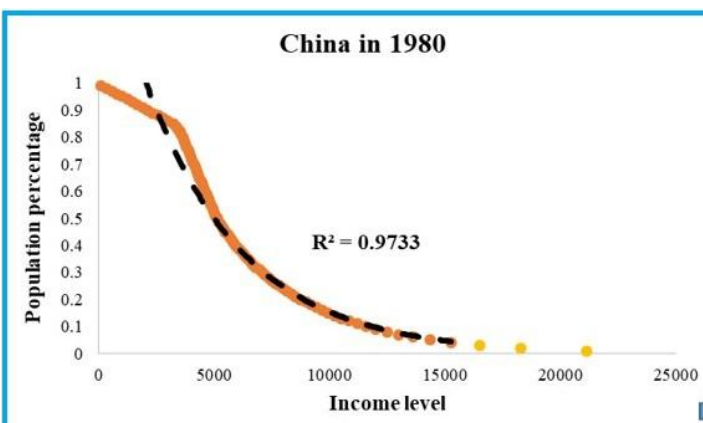

China in 1990

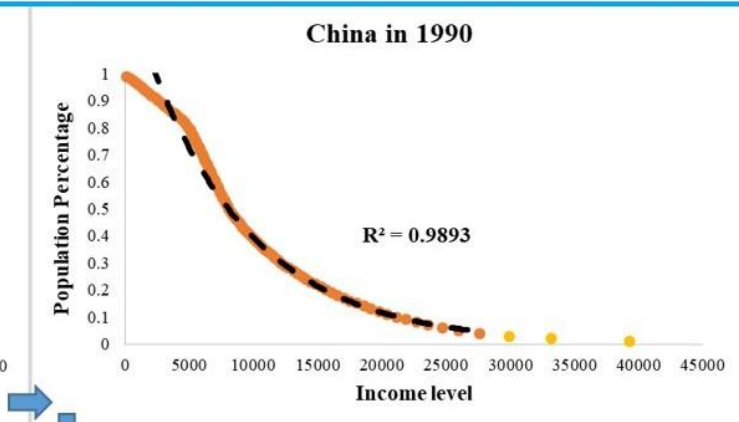

China in 2010

China in 2000
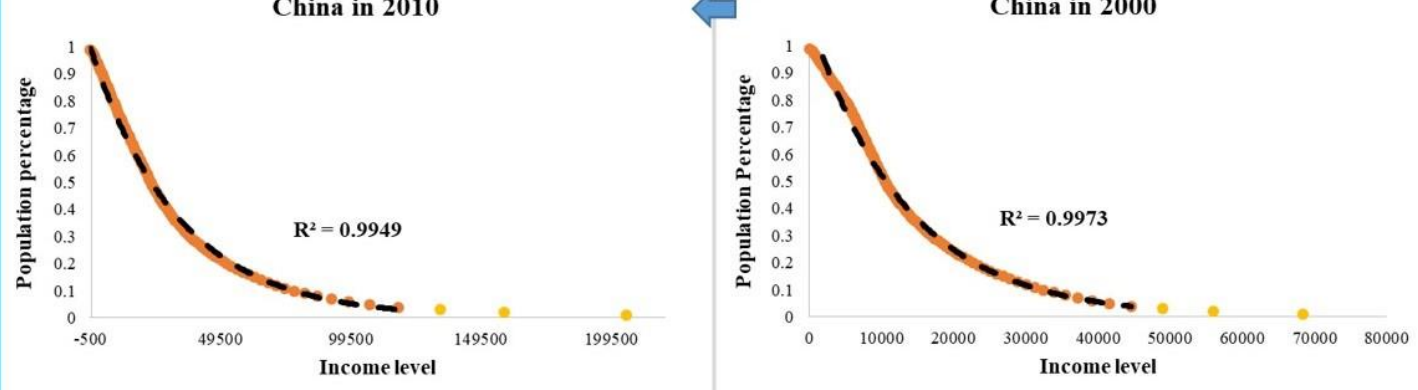

Figure 2: The income structure of China gradually evolved to an exponential distribution after the market-oriented economic reformation ${ }^{8}$.

${ }^{8}$ Data resource for China: http://wid.world/data/ 